\title{
Various Image Compression Techniques: Lossy and Lossless
}

\author{
Manjari Singh \\ Dept. Information \\ Technology \\ LNCT
}

\author{
Sushil Kumar \\ Dept. Information \\ Technology \\ LNCT
}

\author{
Siddharth Singh \\ Chouhan \\ Dept. Information \\ Technology \\ LNCT
}

\author{
Manish \\ Shrivastava, $\mathrm{PhD}$ \\ Dept. Information \\ Technology \\ LNCT
}

\begin{abstract}
Image compression is an implementation of the data compression which encodes actual image with some bits. Thepurpose of the image compression is to decrease the redundancy and irrelevance of image data to be capable to record or send data in an effective form. Hence the image compression decreases the time of transmit in the network and raises the transmission speed. In Lossless technique of image compression, no data get lost while doing the compression. To solve these types of issues various techniques for the image compression are used. Now questions like how to do mage compression and second one is which types of technology is used, may be arises. For this reason commonly two types' of approaches are explained called as lossless and the lossy image compression approaches. These techniques are easy in their applications and consume very little memory. An algorithm has also been introduced and applied to compress images and to decompress them back, by using the Huffman encoding techniques.
\end{abstract}

\section{Keywords}

Image Compression, Huffman Encoding, Lossy, Lossless.

\section{INTRODUCTION}

Image compression is a type of an application for data/image compression in which the basic image gets encoded with the limited bits. To lower the irrelevance and the redundancy of image data is the major target of the image compression is to enable them to get saved or transmit the data in the better form. Image compression is the lowering of the image data size, also with maintaining the required details. The basic objective of the image compression is to show an image in small quantity of bits also the needed content of information is not lost within the actual image. [7]Compression techniques are developd rapidly to compress huge files of datalike images. By the rapid growth of the technology a large quantity of image data should be managed to store those images in the proper manner by the use of effective techniques normally results in the compressing images.

Some algorithms are there which are used to perform these. types of compression in various manners like the lossless and the lossy. The image which is needs to be compressed is a gray scale having the pixel values ranges from 0 to 255 . Compression addressed to the decreasing the number of data to be used to show content of an image, file or the video without decreasing excessively quality of actual data. Also it lowers the quantity of the bits that is needed to save and send the digital media [8].To do compression of something implies that there is a piece of data whose size is forced to decrease. For this the JPEG is a best selection for the digitized-photographs. The system called Joint Photographic Expert Group (JPEG) which is dependent on Discrete Cosine Transform (DCT), have been a very largely used method for compression [9].

Image compression is one of various known techniques in the image processing. This technique may have various implementations and performs a vital role in the effective storage and transmission of the images. The image compression targeted at decreasing the redundancy in the image data to record or sends only few numbers of the samples and by this also a good accession can be reconstruct for the actual image corresponding with the perception of human visual.

To compress images is the major target of this paper by decreasing the number of the bits on the basis of per pixel which is needed to show it and also to lower the time of transmission for the transmission of images and for reconstructing again by the Huffman encoding algorithm.

\section{IMAGE}

An image is a 2-D signal that is processed by human visual systems. [2]These signals that are representing an image are commonly in the form of analog. Although for the storage, processing and the transmission through the computer applications, these signals needed to be converted from the analog form to their digital form. An image or a digital image is usually a 2-Dimensional array of the pixels. In the raw form, the images may cover a huge amount of the memory in the RAM and in the storage, both. Image compression is for reducing the redundancy and irrelevance of image/data to allow them to either store or transmit the data in a better way.

\section{IMAGE COMPRESSION}

Decreasing the irrelevance or redundancy of an image is the fundamental aim of the image compression techniques to provide the facility for storing and transmitting the data in an effective manner .The initial step in this technique is to convert the image from the representation of their spatial domain into a separate type of the representation by the use of few already known conversions and then encodes the converted values i.e., coefficients. This technique allows the huge compression of data as compared to the predictive techniques, though at the cost of the huge computational needs.

Compression is obtained by eliminating any of one or more of the below three fundamental data redundancies:

1. Coding redundancy: This is presented when the less than best (that is the smallest length) code words were used.

2. Inter-pixel redundancy: This results from the correlations between the pixels of an image.

3. Psycho-visual redundancy: This is because of the data which is neglected by human visual-system (that is, visually not required information). 
Image/data Compression attains redundancy for the moreeffective coding:

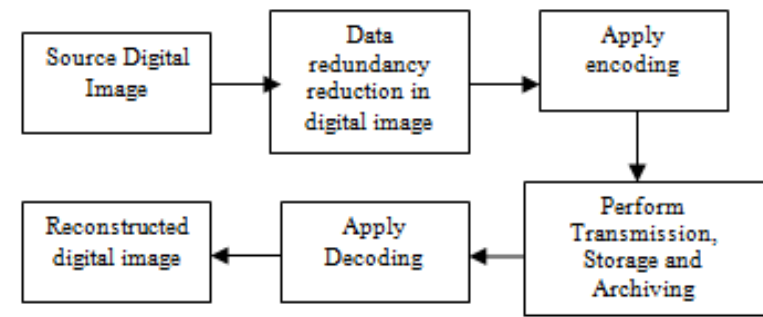

Why do we need compression?

- Increased enough amount of the storage space.

- Decrease the transmission time of an image toget sent on the internet or get downloaded from webpages.

- Multimedia Applications: Desktop Editing

- Image Archiving: Data from Satellite.

- Image Transmission: Data from the web.

\section{TYPES OF IMAGE COMPRESSION}

Image compression can be further classified or divided in two separate types such as lossy compression and losslesscompression. In the lossy compression as its name indicated that it results in the loss of little information. In this technique the compressed image is same as to actual/original uncompressed image yet not exact to the previous one as within the compression process littler information related to the image has been lost. So they are normally applied for the photographs. The very natural example of the lossy compression is a JPEG.

Where are in Lossless compression, it compresses an image by encoding it's all information from the actual file, so in case if the image is get decompressed again, then it will be the exactly same as the actual image. For examples of the lossless technique of image compression are PNG and GIF i.e., GIF only provides 8-bit images. At the time of using a specific format of image compression that basically based on what is being get compressed.

\subsection{Lossless}

In the technique of Lossless compression with the compressing of data that is when get decompressed, will be the same replica of actual data. In this case, when the binary data like the documents, executable etc. are get compressed. This required to be reproduced exactly when get decompressed again. On the contrary, the images and the music also required not to be generated 'exactly'. A resemblance of the actual image is sufficient for the most objective, as far as the error or problems between the actual and compressed image is avoidable or tolerable.

These types of compression are also known as noiseless as they never add noise to signal or image. It is also termed as the entropy coding as it uses the techniques of decomposition/statistics to remove/reduce the redundancy. It is also used only for the some specific applications along with the rigid needs like a medical-imaging. Below mentioned techniques consists in the lossless compression:
1. Huffman encoding
2. Run length encoding
3. Arithmetic coding
4. Dictionary Techniques
a) a)LZ77
b) b)LZ78
c) c)LZW

5. Bit Plane coding

\subsection{Lossy}

In the technique of Lossy compression, it decreases the bits by recognizing the not required information and by eliminating it.[3]The system of decreasing the size of the file of data is commonly termed as the data-compression, though its formal name is the source-coding that is coding get done at source of data before it gets stored or sent[4]. In these methods few loss of the information is acceptable. Dropping non-essential information from the source of data can save the storage area.

The Lossy data-compression methods are aware by the researches on how the people anticipate data in the question. As an example, the human eye is very ensitive to slight variations in the luminance as compare that there are so many variations in the color. The Lossy image compression technique is used in the digital cameras, to raise the storage ability with the minimal decline of the quality of picture. Similarly in the DVDs which uses the lossy MPEG-2 Video codec technique for the compression of the video. In the lossy audio compression, the techniques of psycho acoustics have been used to eliminate the non-audible or less audible components of signal.

\section{BENEFITS OF IMAGE COMPRESSION}

Below are few benefits of the Image compression technique:

- It enables a reliable cost of savings that is included withthe sending of less data on the network of switched telephone in which the cost of call is normally dependent on its duration.

- It is not only to decrease the requirements of storage but also decrease the entire time of execution.

- It decreases the chances of the errors transmission as

- $\quad$ some bits have got transferred.

- It enables a level of the security against monitoring the unlawful Activities.

\section{VARIOUS COMPRESSION ALGORITHMS \\ 6.1 JPEG: DCT-Based Image Coding Standard}

JPEG enables a compression technique which is able to do compressing continuous-tone image or data along with a pixel having depth of the 6 to 24bits with the enough efficiency and speed.[3]

A discrete cosine transform (DCT) describes a fixed series of the data points in the terms of sum of the cosine functions fluctuate at various frequencies. DCTs are vital for various implementations in science and in the engineering area, from the lossy compression of audio for example, MP3and the images for example JPEGin which the small high-frequency elements may be get discarded, to the spectral approached for their numerical solution of the partial differential equations.

By using the cosine instead of the sine functions it is complicated in theseimplementations: for the compression, 
which it returns found that the cosinefunction is so much effective as mentioned here, some functions are required to the exact a typicalsignal, while for the differential equations, the cosines function explains a specific selection of the boundary conditions.

JPEG image compression performs in thepart through rounding off the non-essential bits of the information.[9]Here is an associated trade-off in between theinformation loss and in reduction of size. Various number of the famouscompression techniques have achieved these intuitive differences, consists of those that are used in the music files, video and images. Hence the technique of JPEG's lossy encoding forces to be very prudent with gray-scale portion of the image and be very trivial with color[21].

DCT fragmented the images into the parts of separate frequencies in which less significant frequencies are cancelled through the quantization process and more significant frequencies are used for retrieving the image while the process of decompression.

\subsection{Image Compression by Wavelet Transform}

For various natural signals, the technique of wavelet transform is very efficient tool as compare to the Fourier transform technique. The technique of wavelet transform enables the multi-resolution representation by the use of the set of analyzing functions which are translations and dilations of the some specific functions or wavelets. The technique of wavelet transform found in various forms. Thecomplicated sampled form of wavelet transform enables thevery sophisticated representation; although, it has various limitations also.

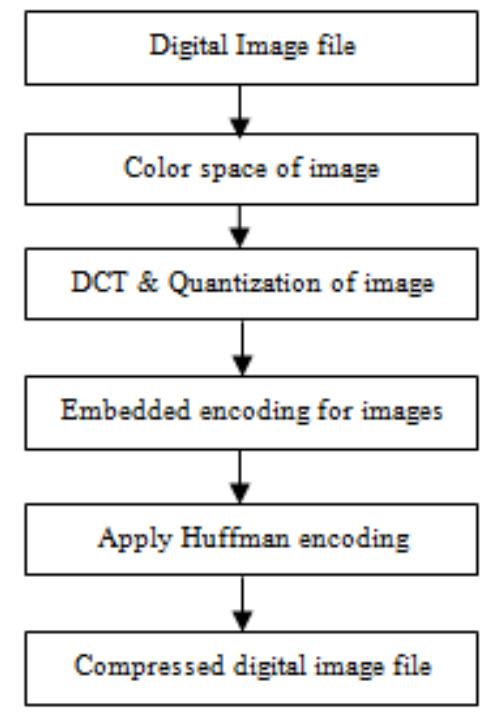

\subsection{Huffman Algorithm}

The general idea in the Huffman encoding algorithm is to allocate the very short code-words to those blocks of input along with the high possibilities and the long code-words are allocated to those which are having the low probabilities.

The Huffman code process is dependent on the two observations mentioned below:

a. Very frequently found symbols will have the shorter codewords as compare to the symbol which found less frequently. b. Two symbols which found least frequently may have the equal length.
TheHuffman code is prepared by combining together twoleast possible characters and that are repeating in this process as far as there is only the one character is remaining. A code-tree is hence prepared and then a Huffman code is generated from thelabeling of code tree.It is the best prefix code that is generated from the set of the probabilities and which has been used inthe different applications of the compression.

These generated codes are ofdifferent length of codewhich is using integral number of the bits. Thisconcept results in a decrease in average length of the code and hence the whole size of the compressed data is become smaller as compare to theoriginal one. The Huffman's algorithm is the first that provides the solutionto the issue of constructing the codes with less redundancy.

\section{LITERATURE REVIEW}

In the paper [1] proposed that a compression technique by the use of the twoloss-less technologies Huffman coding and the Lempel-Ziv-Welch coding for image compression. At first stage of this, a image is get compressed with the Huffman coding that is resulting in the Huffman-tree and a generated Huffman Code-words.In this paper, a technique has been proposed which is called the "Sub-TreesModification of Huffman Coding for Stuffing Bits Reduction and Efficient NRZI Data Transmission".

They basically targeted on transmission of the data and the multi-media compression and also treating this issue as encoding of the compression and the transmission to generate a low bit rate of transmission model which is dependent on the Huffman encoding algorithm. The suggested technique can balance the "0" bit and "1" bitby measuring the chances of mismatch found in the traditional Huffman-tree. Additionally, the suggested techniques also get modified with the transitional-tree within the same ratio of compression [10].

In paper [4]it is presented a vector quantization depend ent technique of image compression [5]. In this paper they also adjusted the encoding for the difference in map between the actual images and then after that it got restored in the VQ compressed variation. It is the experimental results that show there model which is required toenable the additional data, it may considerably enhances the quality of the VQ images compression and also again be compromised based on the difference in map from technique of lossy compression to the technique of lossless compression.

In paper [6] it is represented an ideal technique for the image compression that is referred as the five module method(FMM). In this technique, transforming of every pixel value in the $8 \times 8$ blocks [7] into the multiple of 5 for every array of RGB. Afterwhich the value may be fragmented by 5 to generate the new values that are bit of length for every pixel and which is less in the storage area as compare tothe actual values that is of 8 bits. This paper also represented that theefficiency of the FMM dependent image compression methods.The merits of their techniques are it enables the high PSNR that is peaksignal to the noise ratiothough it is a low CR that is compressionratio. This technique is suitable for the bilevel such as the black andwhite medical images in which pixel in those images isdemonstrated by the one byte ( 8 bit)

\section{CONCLUSION}

A new technique for the image compression that uses the Wavelet-based Image-Coding in the combination with the Huffman encoder has been explained here. This method uses the zero tree architecture ofwavelet-coefficients at the decomposition level of 8 with the Huffmanencoder is a very 
efficiently used that proved in the higherratio of compression and a better PSNR. This paper also helps to the softwaredeveloper to develop new compressed software for the compressing of anyimage as the lossless actual image by the use of Huffman encoding algorithm. Ones it can generate their own frame-work for the compressing any of the imagewith the less difficulties as a space andtime.By the analysis and observation of all these techniques it is found that the lossy compression method enables high compression ratio as compare to the lossless compression method. A Lossy compression technique is used for the more compression ratio and a Lossless compression isused in case of theactual image and for the reconstructed image that are needs to be same.

\section{REFERENCES}

[1] Yu-Ting Pai, Fan-Chieh Cheng, Shu-Ping Lu, and ShanqJang Ruan, "Sub-Trees Modification of Huffman Coding for Stuffing Bits Reduction and Efficient NRZI Data Transmission" IEEE Transactions On Broadcasting, Vol.58,No.2, June 2012

[2] JayavrindaVrindavanam ,SaravananChandran, Gautam K. Mahanti, "A Survey of Image Compression Methods" International Journal of Computer Applications ${ }^{\circledR}$ (IJCA) 2012.

[3] AnilkumarKatharotiya, Swati Patel1 Mahesh Goyani, "Comparative Analysis between DCT \& DWT Techniques of Image Compression" Journal of Information Engineering and Applications Vol 1, No.2, 2011.
[4] Jau-JiShen and Hsiu-Chuan Huang,\| An Adaptive Image Compression Method Based on Vector Quantization,|IEEE, pp. 377-381, 2010.

[5] Suresh Yerva, Smita Nair and Krishnan Kutty,\| Lossless Image Compression based on Data Folding,\|IEEE, pp. 999-1004, 2011

[6] Firas A. Jassim and Hind E. Qassim,\| Five Modulus Method for Image Compression,\| SIPIJ Vol.3, No.5, pp. $19-28,2012$

[7] Vartika Singh "A Brief Introduction on Image CompressionTechniques and Standards" International Journal of Technology and ResearchAdvances Volume of 2013 issue II.

[8] A. Alarabeyyat, S. Al-Hashemi1, T. Khdour1, M. Hjouj Btoush1,S.Bani-Ahmad1, R. Al-Hashemi "Lossless Image Compression Technique Using Combination Methods "Journal of Software Engineering andApplications, 2012.

[9] FouziDouak, RedhaBenzid and Nabil Benoudjit, Color image compression al-gorithm based on theDCT transform combined to an adaptive block scanning, AEUInternational Journal of Electronicsand Communications, vol.65, Issue 1, pp. 16-26, Jan 2011.

[10] Mamta Sharma, "Compression Using Huffman Coding",International Journal of Computer Science and Network Security, Vol.10, No.5,May 2010 